VK Duque Rengel, ME Abendaño Ramírez, AV Velásquez Benavides (2017): “Análisis de los factores de comunicación que inciden en la fidelización de los públicos universitarios". Revista Latina de Comunicación Social, 72, pp. 751 a 764. http://www.revistalatinacs.org/072paper/1190/40es.html DOI: $10.4185 / \mathrm{RLCS}-2017-1190$

\title{
Análisis de los factores de comunicación que inciden en la fidelización de los públicos universitarios
}

\author{
Analysis of communication factors influencing customer \\ loyalty among university students
}

Vanessa Karina Duque Rengel [드] Universidad Técnica Particular de Loja. Ecuador vkduque@utpl.edu.ec

Mónica Elizabeth Abendaño Ramírez [CV] Universidad Técnica Particular de Loja. mabendano@utpl.edu.ec

Andrea Victoria Velásquez Benavides [ $\underline{\mathrm{CV}}]$ Universidad Técnica Particular de Loja. avvelasquez@utpl.edu.ec

\begin{abstract}
s
[ES] Para las organizaciones de cualquier índole, el contar con una cartera de clientes fidelizados se ha cristalizado en el sustento social, económico y de marca, que le permite sostenerse en el contexto cambiante e inestable al que se enfrentan las empresas actualmente. En este escenario nace la presente investigación que es parte de un proceso de mejora continua, cuyo objetivo fue establecer pautas reales para fidelizar a los públicos heterogéneos de las modalidades de estudios a distancia en el Ecuador, a través del planteamiento de estrategias ligadas al mejoramiento del servicio y canales de comunicación, que permitan fortalecer el sentido de pertenencia de los estudiantes universitarios. Metodológicamente fue levantada como estudio de caso en una universidad ecuatoriana. Tomó como objeto de estudio el nivel de pregrado de modalidad a distancia, en donde se analizó los vínculos de dicha institución con sus públicos. El trabajo de campo se basó en una metodología de enfoque mixto, utilizando técnicas de recolección de datos cualitativas (entrevistas) y cuantitativas (encuestas), que permitieron conocer y analizar los puntos de vista de los stakeholders, que permitieron trazar pautas de comunicación estratégica para fidelizar a sus stakeholders.
\end{abstract}

[EN] For all types of organisations, having a portfolio of loyal customers has become the social, economic and brand foundation that allows them to remain standing in the changing and unstable environment currently faced by companies. Based on this scenario, and as part of a process of continuous improvement, this research aims to establish guidelines to build customer loyalty among 
Ecuador's distance-learning undergraduate students, through strategies linked to the improvement of customer service and communication channels, which allow strengthening the sense of belonging of university students. This research is based on the case study of an Ecuadorian university. The relation between this institution and its heterogenous publics is based on the analysis of the opinions of undergraduate distance-learning students. The field work was carried out with a mixed approach that combined qualitative and quantitative data collection instruments (interviews and surveys, respectively), which allowed for the analysis of the stakeholders' points of view and the drawing of strategic communication guidelines to build customer loyalty.

\section{Keywords}

[ES] Universidad; estudiantes; fidelización; servicios; canales de comunicación; pertenencia institucional.

[EN] University; students; loyalty; services; communication channels; institutional affiliation.

\section{Contents}

[ES] 1. Introducción. 2. Método. 3. Resultados y discusión. 4. Conclusiones.

[EN] 1. Introduction. 1.1. Fundamentals of customer loyalty building. 1.2. Loyalty matrix. 1.3. Customer loyalty indicators and categories. 1.4. Benefits of loyalty. 2. Methods. 2.1. Scope. 2.2. Techniques. 2.2.1. Direct observation. 2.2.2. Interview. 2.2.3. Survey. 3. Results and discussion. 4. Conclusions. 5. References.

Traducción de CA Martínez-Arcos

(Doctor en Comunicación, Universidad de Londres)

\section{Introducción}

Las Instituciones de Educación Superior han sido creadas con la finalidad de dar respuesta a una necesidad de las comunidades, específicamente para preparar individuos en diferentes áreas del saber, que sirvan a la sociedad como entes productivos, con un alto enfoque humanista y con sólida base científico- técnica. Por ello se afirma que: "El cultivo de la ciencia y las humanidades se encuentra en otro plano de la existencia colectiva." (Revista EKOS, 2009: 126)

En el país la educación superior es un campo en el que participan instituciones de diverso perfil y que a su vez ofertan un sin número de carreras en diferentes modalidades de estudio. Uno de los inconvenientes comunes a los que se enfrentan estas organizaciones son la deserción estudiantil, "cuyas consecuencias, de tipo social y económico afectan directamente al entorno familiar, a la comunidad académica y al país, y no se limitan solo al Ecuador.” (Fernández, 2014:34)

De acuerdo a diversos estudios realizados en la última década, la deserción estudiantil es mayor en la modalidad de estudios a distancia, especialmente en los primeros años, entre los factores que se ha identificado como causas de deserción está: el método de estudios, problemas económicos, falta de tiempo para estudiar, entre otras. Un ejemplo de esta realidad se muestra en la figura 1, tomado del "Estudio de índice y causas del abandono temprano de los estudios universitarios en modalidad a distancia", en el cual se evidencia que más del 50\% de la población estudiantil abandonan sus estudios en la etapa inicial. 


\section{RLCS, Revista Latina de Comunicación Social, 72 - Páginas 751 a 764 Investigación | DOI: 10.4185/RLCS, 72-2017-1190| ISSN 1138-5820 | Año 2017}

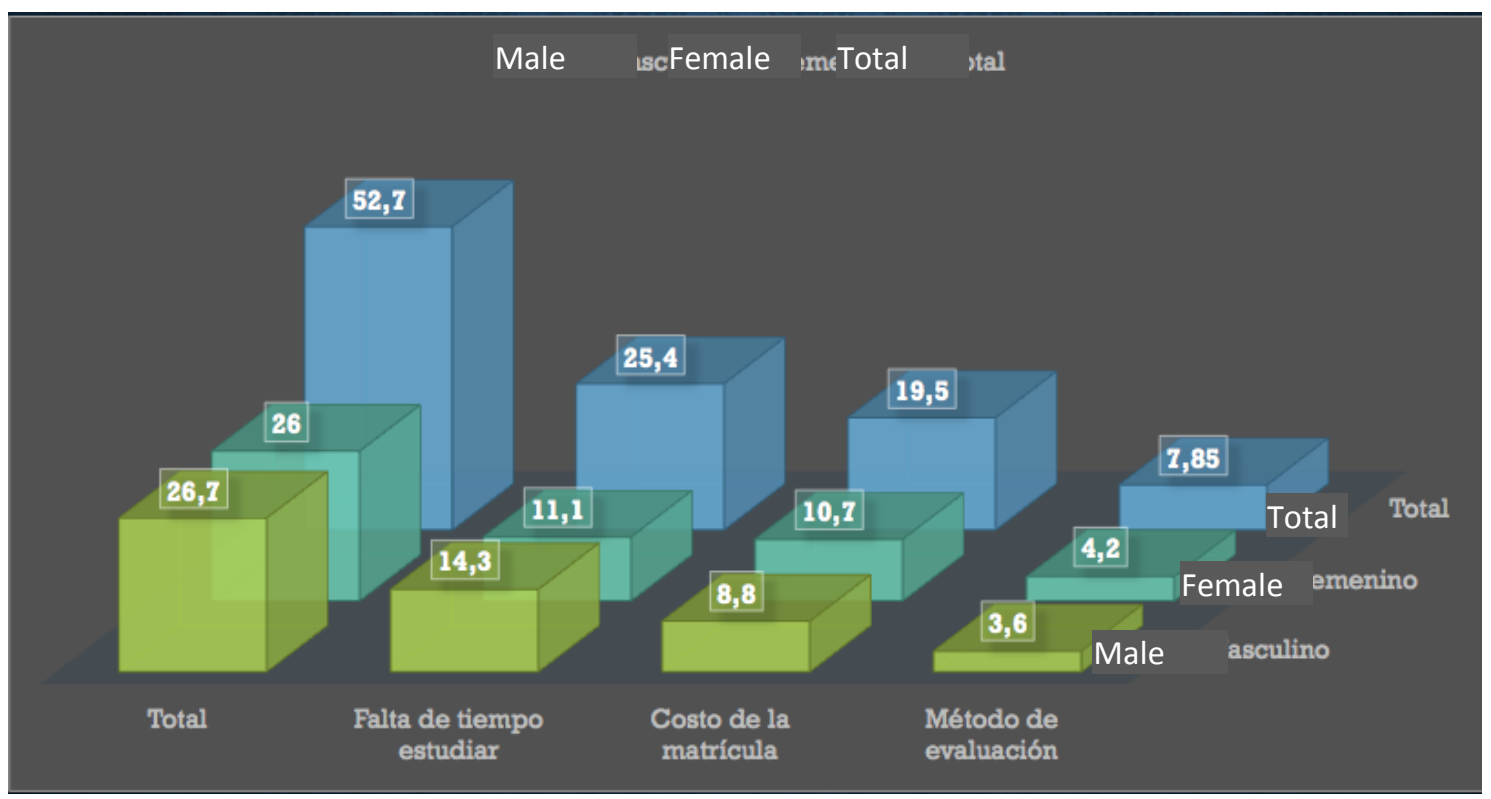

Figura 1: Índice de deserción estudiantil en una Universidad Ecuatoriana Tomado de Buele, Ramón \& Sánchez, 2013: 38

Frente a este escenario, la fidelización de los públicos puede contribuir a disminuir los niveles de deserción estudiantil. Así se plantea esta investigación desarrollada como caso de estudio de una universidad ecuatoriana de modalidad a distancia, cuyo objetivo fue establecer los factores de comunicación que inciden en la fidelización de los públicos.

Entendiéndose como fidelización a "una actitud positiva, que supone la unión de la satisfacción del cliente (formada por elementos racionales, afectos y comportamientos) con una acción de consumo estable y duradera." Alcaide (citado por Agüero y Collado, 2014: 3)

Cubrir las necesidades de los clientes, resulta hoy necesario, a fin de proporcionarles a dichos públicos productos y servicios que estén a su altura y acorde a sus pretensiones, estos es lo que fomenta la fidelización.

Según el Instituto de Estudios Superiores de Monterrey (2011), la fidelización comprende "las acciones de una empresa dirigidas a configurar relaciones estables con clientes a largo plazo, para crear un sentimiento de pertenencia del cliente hacia la organización, y en consecuencia hablarán de ella de forma positiva".

A partir de esta aproximación teórica, la fidelización de clientes, en el contexto actual al que se enfrentan las organizaciones, puede consolidarse en una ventaja competitiva sostenible para la empresa frente a la industria.

Fuentes (2015) reflexiona sobre el nuevo entorno al que se enfrentan las empresas y destaca que:

La cultura de servicio, evoluciona de acuerdo a la dinámica del entorno; que el mercado y el perfil del cliente cambien. Esta dinámica transformó el concepto de la venta de transacciones, a una venta relacional; donde la construcción de vínculos con el cliente es prioritaria y se convierte en un elemento diferenciador para alcanzar una mayor competitividad en el 
mercado. En este nuevo escenario la comunicación toma un papel protagónico, porque es a través de su gestión donde se posibilita la construcción de relaciones y de vínculos. (pág.17)

Fidelizar no solo implica retener clientes, sino que estos públicos pueden constituirse en la carta de presentación frente a los potenciales clientes, a través de la recomendación.

Es así que, las organizaciones de toda índole, como en el presente caso de estudio, del ámbito educativo, deben volcar sus esfuerzos en alcanzar altos niveles de satisfacción en sus clientes, que es la base para garantizar la fidelidad de los mismos. Es por ello, que la empresa debe mostrar su interés y compromiso con sus públicos.

No obstante, establecer vínculos con los stakeholders, es una de las tareas primordiales de la comunicación, para ello, parte de la gestión de la comunicación y marketing empresarial debería estar dirigido en potenciar y optimizar el servicio a los clientes, que consiste en lograr que el público de interés y/o consumidor, se convierta en un cliente fiel a la organización.

\subsection{Fundamentos de la fidelización}

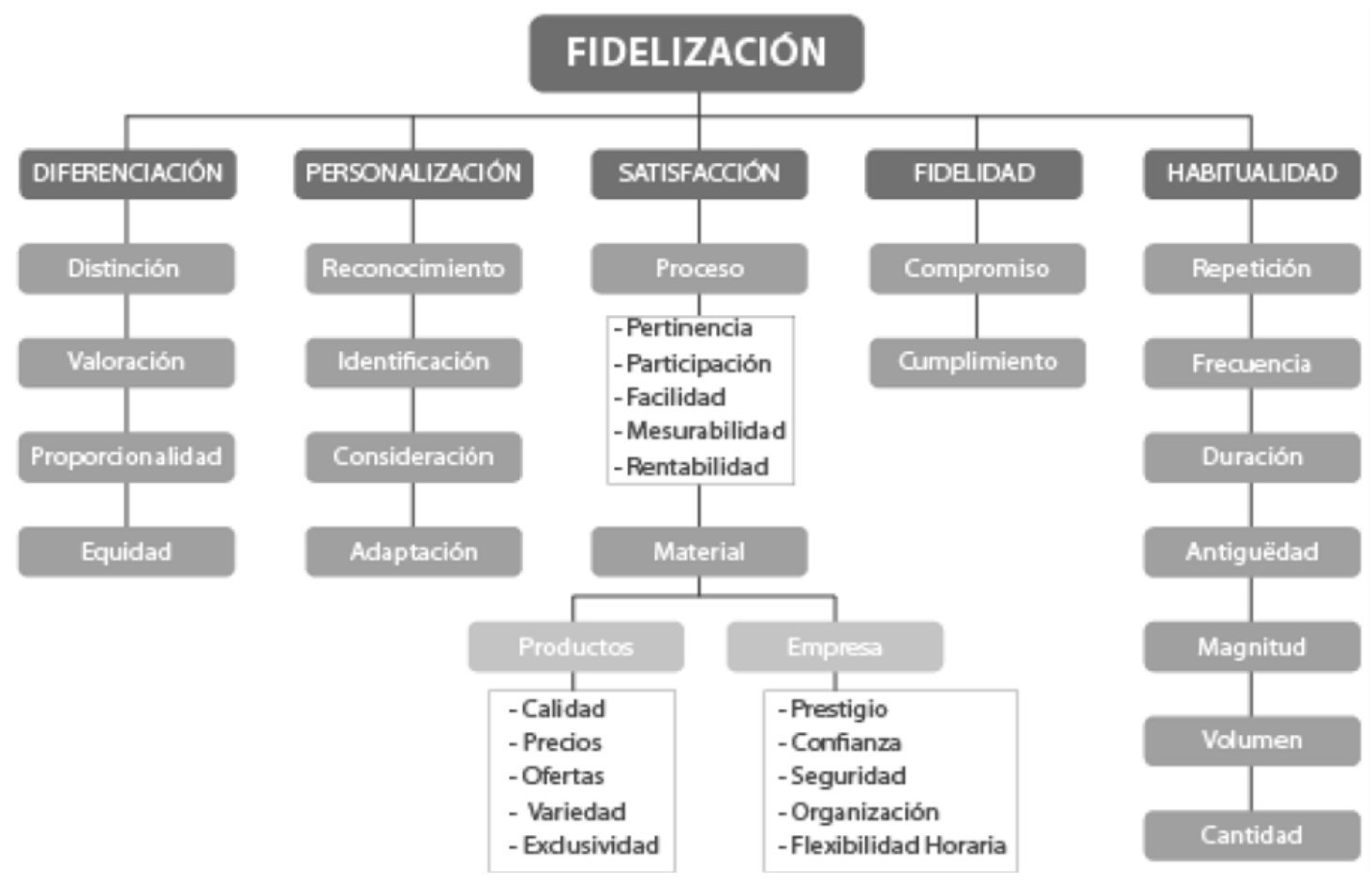

\section{Figura 2: Fundamentos de fidelización de clientes}

Adaptado del Instituto Tecnológico de Monterrey. (s.f.)

Las emociones se constituyen en una de las herramientas estratégicas para trabajar la fidelización de los clientes. Así lo corrobora el siguiente esquema expuesto en la figura 1, en la que aborda cinco aspectos a tener en cuenta en el proceso de fidelización, que son la diferenciación, personificación, 
satisfacción, fidelidad y habitualidad, cada uno de estos aspectos integra una serie de acciones a cumplir para alcanzarlo.

\subsection{Matriz de fidelización}

Schnarch (2011) propone una interesante matriz de fidelización que permite clasificar y medir el grado de satisfacción y relación de sus clientes, que se muestra a continuación:

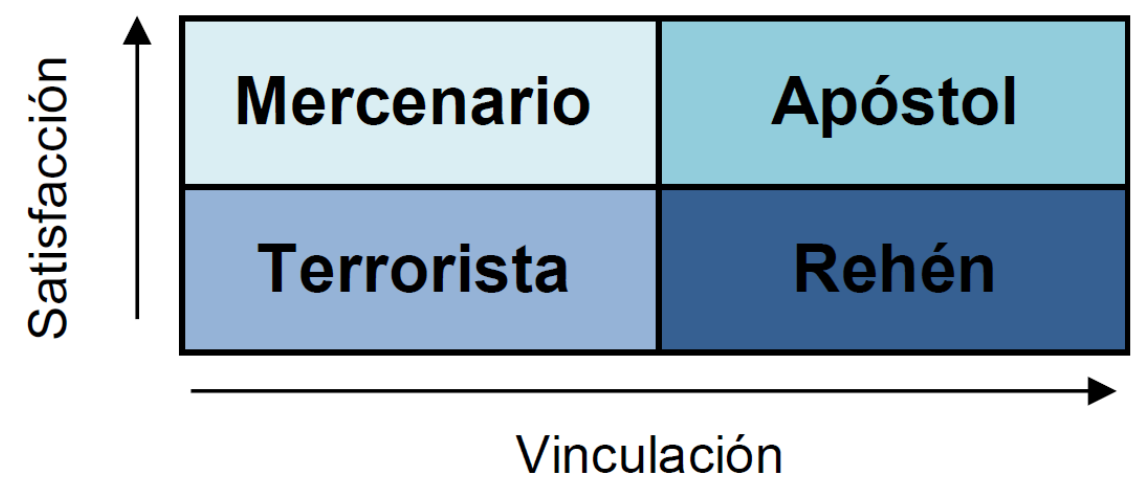

Figura 3: Matriz de fidelidad

Tomado de Schnarch, 2011: 72

El autor explica el significado de cada uno de los términos empleados en la matriz:

- Terroristas: aquellos que hablan mal de la empresa porque no están satisfechos y tampoco hemos logrado establecer vínculos con ellos.

- Mercenarios: que están satisfechos, pero no hay relación establecida con ellos, por lo tanto a cualquier mejor oferta de la competencia se van.

- Rehenes: clientes con los cuales se ha establecido relaciones, pero no están satisfechos.

- Apóstoles: hablarán bien y nos recomendarán, ya que están satisfechos y tenemos vínculos estrechos que incluso crean barreras contra el cambio.

Establecer en cuales, de estos cuadrantes, se ubican los estudiantes de la Universidad, es sin duda necesario para conocer su nivel de satisfacción del servicio educativo que ofrece la institución, y poder plantear estrategias idóneas, que permitan llegar a todos los púbicos a identificarse como apóstoles.

\subsection{Indicadores y categorías de fidelización}

Artel (citado por Romero, 2014) define los indicadores y categorías de fidelización de la siguiente manera (pág.17):

- Fidelidad exclusiva.

- Fidelidad que prevé compras de más marcas al mismo tiempo.

- Fidelidad a las marcas alternadas.

- La infidelidad: limitadas compras pero con una actitud positiva hacia el producto.

Así mismo explica que, la fidelización se divide en tres partes que son: 
- La penetración: el número de clientes que adquirieron la marca en un tiempo determinado, comparado con el número de clientes que compraron el mismo producto solo que a otras empresas durante el mismo período determinado de tiempo.

- La frecuencia: El número de veces que compra un cliente la marca comparado con el número de veces que un cliente compra en otras marcas durante un mismo período de tiempo.

- La exclusividad: El número de clientes que han comprado una marca comparada con número de clientes que han comprado otras marcas en un mismo período de determinado de tiempo.

El poder conocer estos factores en la relación entre el cliente y la organización, permite determinar en qué sitial están los públicos, cuál es su nivel de compromiso con la organización. Así mismo, brinda información sobre el estado actual del servicio que se ofrece y como se lo está percibiendo.

\subsection{Beneficio de fidelización}

De acuerdo a Vieites, (2012) tener satisfechos a los clientes supone, no sólo la posibilidad de que éstos repitan la compra en el negocio, sino una serie de beneficios añadidos para la empresa que se enumeran a continuación:

- Son prescriptores. Los clientes fieles suelen recomendar el establecimiento por lo que se convierten en los mejores prescriptores.

- Mejora continua. Los clientes fidelizados conocen bien el negocio y poseerán un mayor nivel de confianza por lo que estarán en mejores condiciones para aportar sugerencias y mejoras al negocio.

- Mayor conocimiento de los clientes por parte de la empresa. Cuanto más se relaciona la empresa con un cliente más y mejor se le conoce y por lo tanto se podrá adaptar mejor a sus necesidades o preferencias. Creando un bucle de mejora.

- Rentabilidad para la empresa. Es más económico fidelizar un cliente, que captar otro nuevo. Estos clientes además son menos sensibles a las subidas de precios porque valoran el servicio que se les presta y por lo tanto estarán dispuestos a pagar más.

- Aumento de ventas: Resulta más fácil vender un nuevo producto a un cliente actual que a un cliente nuevo que no conoce el negocio. Además, los clientes fieles es más probable que demanden nuevos productos o servicios de la empresa.

\section{Metodología}

En la presente investigación se aplicó una metodología mixta, es decir, con técnicas cualitativas y cuantitativas, con el objeto de conocer la percepción de distintos aspectos institucionales de la Universidad y su modalidad de estudios a distancia.

El análisis cuantificable se realizó a través de la técnica de la encuesta, conformada por preguntas cerradas, de percepción y ponderación, que fueron aplicas a la muestra escogida del universo a investigar.

La combinación de los métodos inductivo y deductivo, al ser procedimientos que van de lo particular a lo general y viceversa, se utilizaron para la observación directa y análisis documental, para posteriormente contrarrestar el diagnóstico de la situación y las conclusiones finales. Así mismo se utilizó la entrevista a profundidad a directivos de la institución. 


\subsection{Alcance}

En el estudio se aplicó un alcance de tipo exploratorio - descriptivo.

Exploratorio para establecer y destacar los aspectos fundamentales de una problemática determinada y encontrar los procedimientos adecuados para diagnosticar correctamente la situación actual de la gestión de comunicación y el accionar de fidelización de los públicos.

Descriptivo con el propósito de la adquisición de datos objetivos, precisos y sistemáticos que se usarán en el análisis de datos e interpretación de resultados.

Todo ello permitió un entendimiento más claro y objetivo del tema, así como, el planteamiento de base de la propuesta.

\subsection{Técnicas}

Las técnicas utilizadas en la presente investigación son:

\subsubsection{Observación directa}

Esta técnica permitió realizar una observación profunda del caso de estudio, estableciendo una relación directa entre el objeto de estudio y el investigador; que facilitó la comprensión del contexto de estudio.

\subsubsection{Entrevista}

Se llevaron a cabo tres entrevistas a profundidad dirigidas a directivos que lideran instancias directamente vinculadas con los públicos de la modalidad a distancia.

\subsubsection{Encuesta}

Se aplicó una encuesta online de 10 preguntas de ponderación y percepción a una muestra de 395 estudiantes, resultado de la fórmula que se muestra a continuación:

$$
\begin{aligned}
& \mathrm{n}=\mathrm{N} \\
& \mathrm{E} 2(\mathrm{~N}-1)+1 \\
& \mathrm{n}=\text { muestra } \\
& \mathrm{N}=\text { población } \\
& \mathrm{E} 2=\text { error al cuadrado }(5 \%) \\
& 29519 \text { estudiantes }
\end{aligned}
$$$$
\mathrm{n}=\frac{29519}{0,0025(29519-1)+1}=0,0025665 \text { o } 1,7 \%=395
$$

395 estudiantes

La encuesta abarcó tres dimensiones: servicio al cliente, canales de comunicación, percepción y atributos de imagen. 


\section{RLCS, Revista Latina de Comunicación Social, 72 - Páginas 751 a 764 Investigación | DOI: 10.4185/RLCS, 72-2017-1190| ISSN 1138-5820 | Año 2017}

\section{Resultados y discusión}

\section{- Encuesta}

En el desarrollo de la investigación se consideró necesario establecer las razones por las que los estudiantes optan por esta modalidad, pues este es un factor determinante para conocer las potencialidades de fidelización.

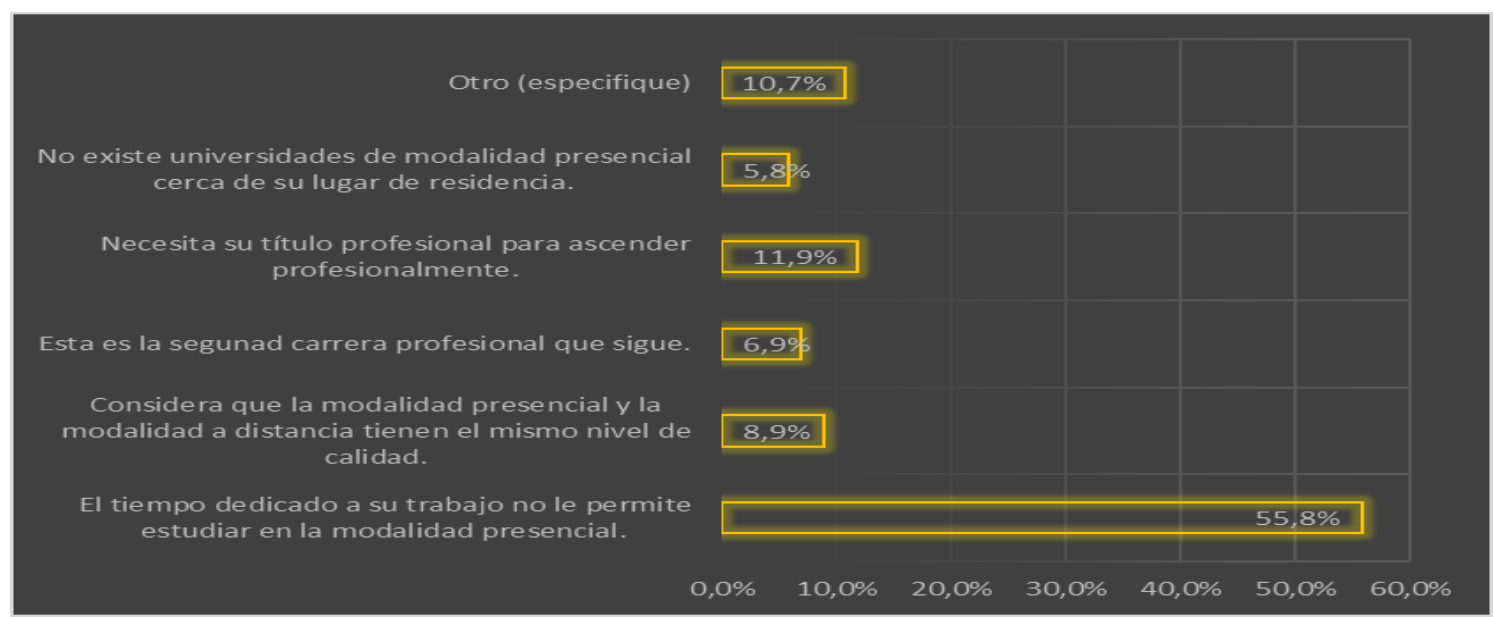

Figura 4: Razones de elección de la modalidad a distancia.

Más de la mitad de la población encuestada, un 55, 69\% eligió estudiar en la modalidad a distancia porque el tiempo dedicado a su trabajo no le permite estudiar en la modalidad presencial, seguido de las personas que necesitan un título profesional para ascender profesionalmente con un $11.89 \%$. Aquellas personas que se inclinan por la variable otros, especifican en su gran mayoría que su tiempo es limitado porque lo dedican al cuidado de su familia.

A continuación se exponen los resultados de las 3 dimensiones abordadas:

\section{Servicio al cliente}

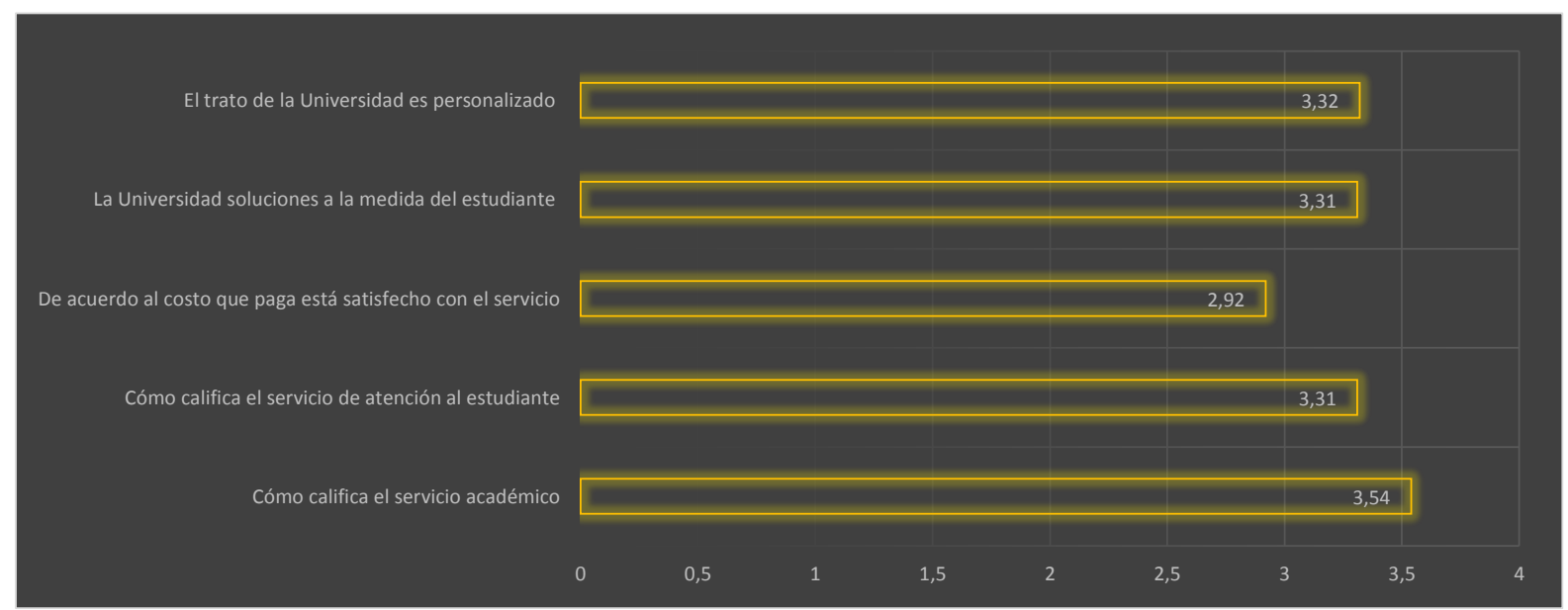

Figura 5: Índice de satisfacción del estudiante de la MAD UTPL. 


\section{RLCS, Revista Latina de Comunicación Social, 72 - Páginas 751 a 764 Investigación | DOI: 10.4185/RLCS, 72-2017-1190| ISSN 1138-5820 | Año 2017}

En esta interrogante de la encuesta se evaluó el nivel de satisfacción de los estudiantes en cinco aspectos, ponderados de 1 a 5, obteniendo una media de 3.28/5. En conclusión se supera más de $50 \%$ del total de la calificación, ello no implica que no debe mejorarse la evaluación de estos aspectos.

\section{Canales y herramientas de comunicación}

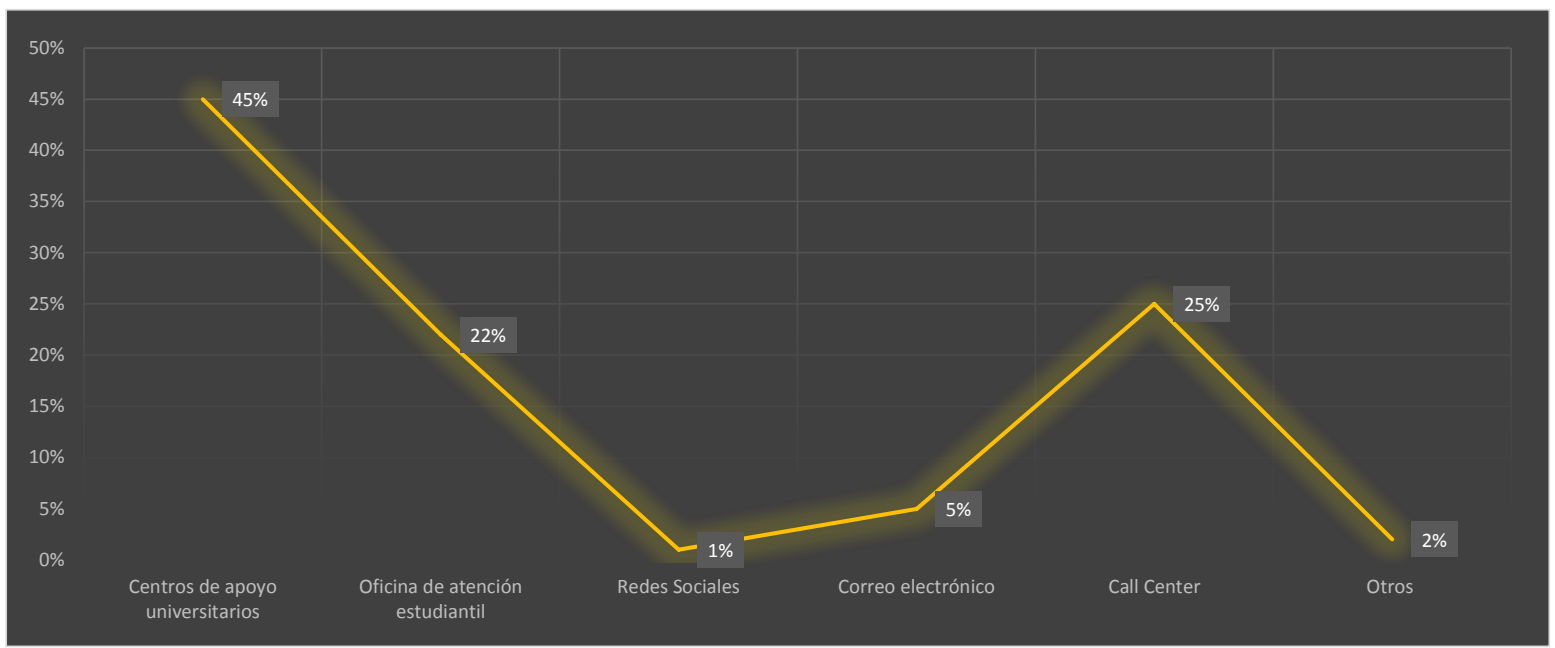

Figura 6: Identificación de los canales de comunicación para trámites efectivos.

Los centros de apoyo universitarios son los canales a través de los cuales los estudiantes realizan sus trámites académicos, con un porcentaje de 43,31\%. Así mismo, el call center con un 25,06\% y el balcón de servicios estudiantiles con un $21,51 \%$ son los que le siguen en utilidad a los públicos. El correo electrónico y las redes sociales son el canal de menos uso en el tema de servicios administrativos, con un 4,81 y $1.01 \%$, respectivamente. En cuanto a otros canales los encuestados no señalas cuales.

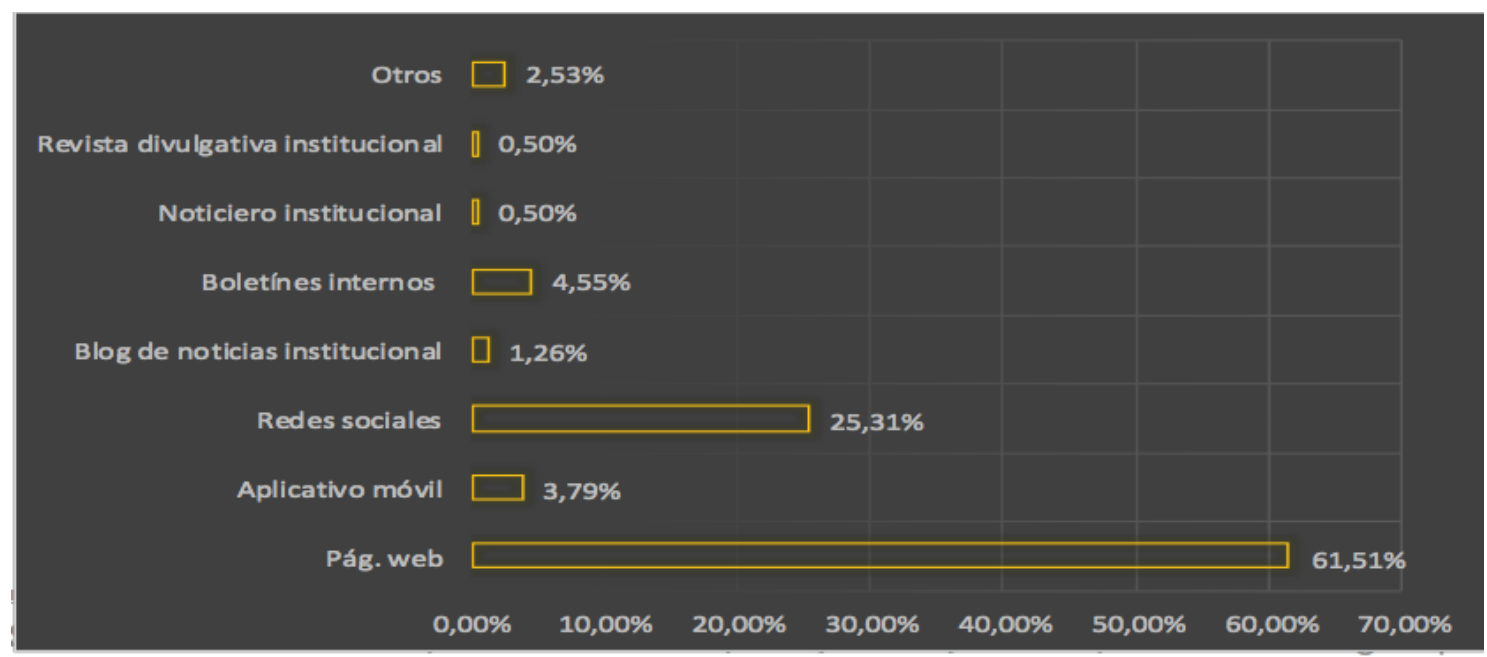

Figura 7: Identificación de canales de carácter informativos. 


\section{RLCS, Revista Latina de Comunicación Social, 72 - Páginas 751 a 764 Investigación | DOI: 10.4185/RLCS, 72-2017-1190| ISSN 1138-5820 | Año 2017}

$61.51 \%$ de la población estudiantil se informa del acontecer universitario a través del portal web de la Universidad, lo que muestra una gran aceptación de este canal. Seguidamente se encuentran las redes sociales con una $25.31 \%$ de aceptación. Existe un bajo porcentaje que utilizan el aplicativo móvil y el boletines de comunicación internos para informarse, con un porcentaje de 4.75 y $3.79 \%$, lo que implica que no están cumpliendo con el fin para el que fue creado.

\section{Imagen y atributos}

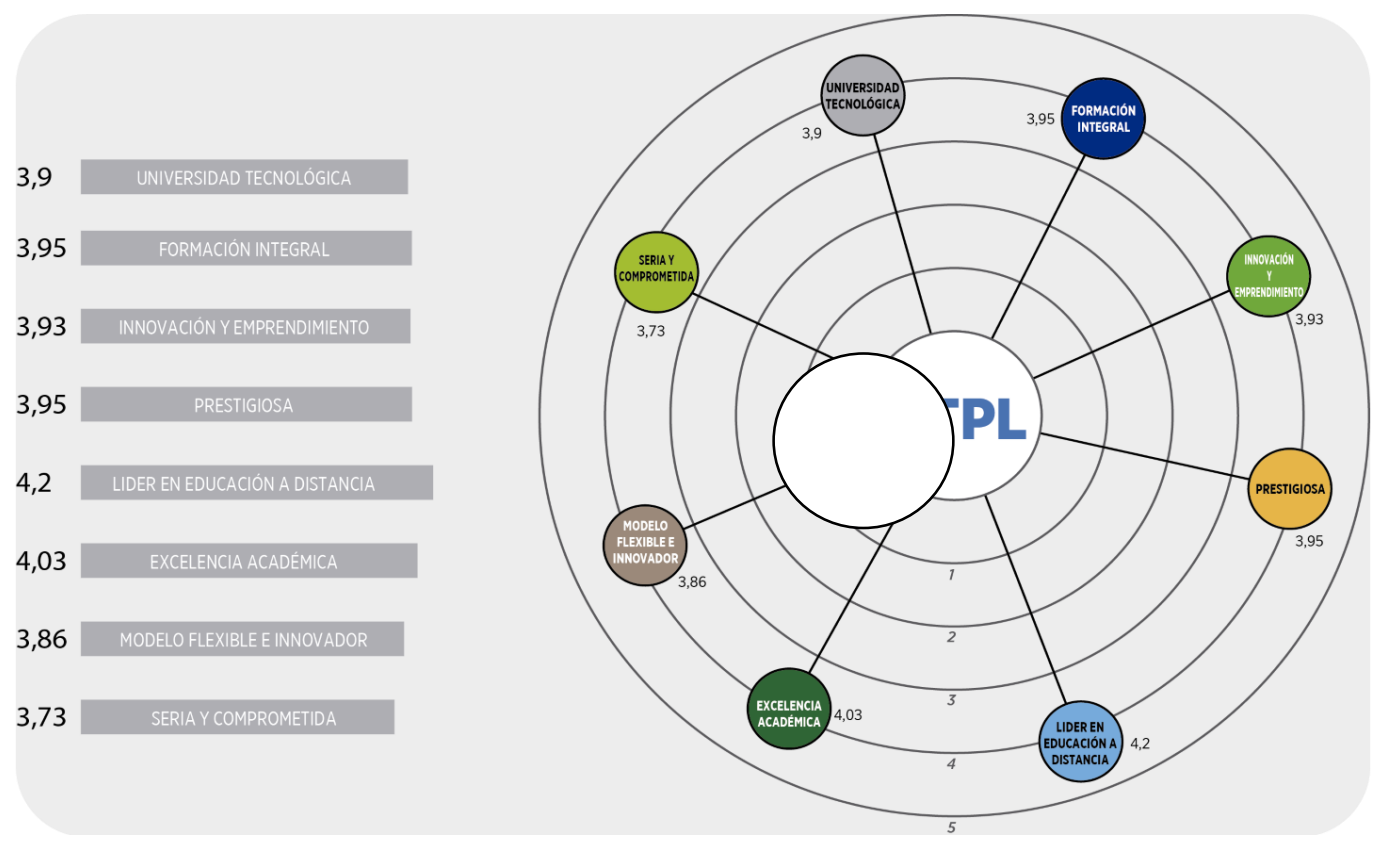

Figura 8: Ponderación de atributos (Constelación de atributos).

Para fines de la investigación se consideró necesario el conocer como los estudiantes de la Universidad la identifican con los atributos a continuación descritos citados en la figura 8, en la que destacan "líder en educación a distancia" y "excelencia académica".

\section{Entrevista}

A fin de establecer el criterio de la deserción estudiantil y la importancia de la comunicación en la fidelización de las audiencias universitarias, se desarrolló entrevistas en profundidad a tres directivos universitarios, de las cuales se extrajeron las siguientes conclusiones:

- El perfil de los estudiantes de la modalidad a distancia está caracterizado por ser personas que tienen responsabilidades laborales y/o familiares, en su gran mayoría son empleados de empresas privadas. Su edad abarca desde los 17 años hasta los 68, siendo el mayor porcentaje desde los 17 hasta 21 años, quienes se caracterizan por su aspiración de insertarse en el ámbito laboral tempranamente. El mayor porcentaje de apertura de la modalidad a distancia está geográficamente en la región sierra.

- Es un modelo académico de la modalidad a distancia integra elementos centrados en el estudiante, que son: área de profesores y tutores, función tutorial, recursos y materiales físico y digitales, con un soporte tecnológico. 
- Se considera necesario que el acompañamiento al estudiante por parte del profesor autor y tutor sea más riguroso y personalizado.

- En los últimos años se ha hecho un esfuerzo porque el servicio de carácter administrativo que da soporte a las necesidades del estudiante, sea cada vez más ágil, sistematizado y automatizado, de tal forma que el estudiante no tenga que emplear tiempo de ir a un centro universitario para un trámite, sino que lo puede hacer en línea.

- Institucionalmente se considera que la principales razones de deserción estudiantil son: problemas económicos; dificultad de organización de tiempo entre responsabilidades estudiantiles, familiares y laborales; y deficiencia en conocimientos académicos base, previo al ingreso a la universidad.

- Se debe crear canales de comunicación que esté más al alcance del estudiante de modalidad a distancia y potenciar y mejorar la efectividad de los canales ya existentes, tomando en cuenta el perfil, los requerimientos y necesidades de los públicos.

- La gestión de la comunicación actualmente centra el mayor porcentaje de sus esfuerzos en la captación de nuevos estudiantes, y las estrategias más utilizadas son la comunicación y difusión masiva, marketing relacional y marketing directo.

- Se debe implementar planes de fidelización dirigido a los públicos de la modalidad a distancia.

\section{Conclusiones}

- El modelo académico de la Universidad investigada, cuenta con un alto porcentaje de aceptación por parte de los estudiantes. Sin embargo, estos públicos tardan en adaptarse al modelo, y esta variable se constituye en un factor importante de deserción estudiantil. Ante ello, una estrategia de fidelización sería el desarrollar procesos de inducción más profundos, en los que se demuestren que el estudiante ha adquirido una experticia en el modelo. Además se debe prever el incluir, dentro de esta inducción, aspectos relacionados con la identidad y cultura institucional a fin de crear sentido de pertenencia desde la primera etapa de la vida estudiantil.

- La modalidad de estudios a distancia, se caracteriza por poseer una gama de herramientas tecnológicas dirigidas a los estudiantes, que se desconocen y/o no se usan, provocando que se desaproveche las bondades que brindan estos elementos en el proceso de enseñanza aprendizaje y comunicación, por ello debe trabajarse en socialización y difusión efectiva de los mismos.

- El mayor porcentaje de estudiantes que optan por la modalidad de estudios a distancia es una población joven (de 18 a 27 años). Sin embargo, existen varios perfiles dentro de estos públicos que responde a diferentes realidades. Por tal motivo, es pertinente definir dichos públicos y establecer estrategias para solventar las necesidades de cada perfil.

- El nivel de satisfacción de los estudiantes de la modalidad a distancia de la Universidad, objeto de estudio, se ha evaluado sobre cinco aspectos (el trato, soluciones a la medida del cliente, el costo que paga, el servicio administrativo y el servicio académico) obteniendo un nivel de satisfacción del 66\%, lo que evidencia, que debe evaluarse y mejorarse algunos procesos y servicios, que deben estar acordes al perfil y necesidades del estudiante.

- El accionar básico de la fidelización del cliente está relacionada con la atención que este recibe. La percepción de valores que dichos públicos tienen de la organización constituye una pieza clave en la gestión empresarial. Al ser la fidelización una factor determinante en la deserción y abandono del público frente a la empresa, las universidades deben considerar 
como prioritario el trabajar planes destinados a crear sentido de pertenencia, dirigido a cada uno de los perfiles estudiantiles, a través de un accionar estratégico - táctico, que abarque las áreas vinculadas con la modalidad de estudios, incluida el área o departamento de comunicación y marketing.

- Los canales de comunicación (informativos) con mayor acogida por parte de los estudiantes de la modalidad a distancia son: el portal web y las redes sociales, razón por la cual debe aprovecharse estratégicamente este aspecto para comunicar los temas más relevantes con los que se desea llegar a los estudiantes. Además debe potenciarse los canales restantes.

- Los atributos de imagen de la Universidad, (Prestigiosa, seria y comprometida, Líder en educación a distancia, Universidad tecnológica, Excelencia académica, Modelo flexible e innovador, Formación integral, Innovación y emprendimiento) son identificados por los estudiantes con una media general de 3,94/5, que equivale al 78,8\%, y permite evidenciar que el posicionamiento de imagen a pesar de ser bastante aceptable, tiene baja influencia al momento de la deserción en los estudiantes.

\section{Bibliografía}

Agüero, L. y Callado, J. (2014): Estrategia de fidelización de clientes. Tesis de grado. Cantabria, España: Universidad de Cantabria. Recuperado de: http://repositorio.unican.es/xmlui/bitstream/handle/10902/4474/\%5B2\%5D\%20Ag\%C3\%BCero\%20 Cobo\%20L.pdf?sequence $=1$

Álvarez, A. (2011). Medición y Evaluación en Comunicación. España: Instituto de Investigación en Relaciones Públicas (IIRP).

Buele, M., Ramón, L. \& Sánchez, C. (2013): Estudio de índice y causas de abandono temprano de los estudios universitarios de modalidad en distancia. Boletín REDIPE. Revista de la Red Iberoamericana de Pedagogía. Recuperado de: https://issuu.com/redipe/docs/boletin_830/38

Capriotti, P. (1992). La Imagen de Empresa. Estrategia para una comunicación integrada. Barcelona, España: El Ateneo.

Capriotti, P. (2013). Planificación estratégica de la imagen corporativa. ( $4^{\circ}$ ed.). Málaga, España: Instituto de Investigación en Relaciones Públicas.

Costa, J. (2005). Master DirCom: Los profesores tienen la palabra. La Paz - Bolivia: Joan Costa y Grupo Editorial Design.

Costa, J. (2012): El DirCom de hoy: Dirección y Gestión de la Comunicación en la nueva economía. Barcelona: CPC Editor.

Costa. J. (2015): El paradigma DirCom: El nuevo Mapa del Mundo de la Comunicación y el management estratégico global. Barcelona: Costa Punto Com Editor.

Fernández, X \& Silva, E. (2014): Cuadernos de contrato social por la educación: Deserción estudiantil universitaria en el primer semestre. El caso de una institución de educación superior. Contrato Social por la Educación: Ecuador. Recuperado de: http://uide.edu.ec/media/1365/10.pdf 
Fuente, S. (2015): Módulo Comunicación de Marketing. Quito, Ecuador: Universidad de las Américas: Master DirCom.

García, J. (2009). Comunicación \& Marketing. España: LabCom Books. Recuperado de: http://www.livroslabcom.ubi.pt/pdfs/20110817-sixto_garcia_marketing_2010.pdf

Instituto Tecnológico y de Estudios Superiores de Monterrey, Universidad Virtual. (2011): MK152CRM (Customer Relationship Management) como estrategia de mercadotecnia. Diplomado en Mercadotecnia. México.

Ménsen, V. (2011): Fidelización de clientes: concepto y perspectiva contable. Artículo, Costa Rica: Revista digital de la Escuela de Administración de Empresas del Tecnológico de Costa Rica. Recuperado de: http://revistas.tec.ac.cr/index.php/tec_empresarial/article/view/586

Ménsen, V. (2011). Fidelización de clientes: concepto y perspectiva contable. Artículo, Costa Rica: Revista digital de la Escuela de Administración de Empresas del Tecnológico de Costa Rica.

Moreno, C. y Cerro, S. (2009). Valores empresariales: de la teoría a la práctica. Cataluña: España: Generalidad de Cataluña Departamento de Economía y Finanzas. Recuperado de: http://www.url.edu/ethos/wp-content/uploads/2012/11/VALORES-EMPRESARIALES.pdf

Muriel, M. \& Rota. G. (1980). Comunicación Institucional: Enfoque Social de las Relaciones Públicas. Quito: Editora Andina.

Ocampo, M. (2011). Comunicación Empresarial. Plan estratégico como herramienta gerencial y nuevos retos del comunicador en las organizaciones. Bogotá ECOE Ediciones.

Reinares, P. y Ponzoa, J. (2004): Marketing Relacional: un nuevo enfoque para la seducción y fidelización del cliente. ( $2^{\circ}$. ed.). Madrid, España: Prentice Hall Financial Times.

Ritter, M. (2012). Cultura Organizacional. Buenos Aires: La Crujía Ediciones.

Ritter, M. (2013). El Valor del Capital Reputacional: por qué la opinión que el público tiene de su empresa es un activo estratégico. España: Ritter and Partners Comunicación Estratégica.

Romero, G. (2014): Plan de marketing relacional para fidelizar a los clientes de la Escuela Rusa de Ballet en el cantón Samborondón. Tesis de grado. Guayaquil, Ecuador: Universidad Católica de Santiago de Guayaquil. Recuperado de: http://repositorio.ucsg.edu.ec/bitstream/3317/2472/1/T-UCSG-PRE-ESP-CIM-51.pdf

Schnarch, A. (2011): Marketing de fidelización: Cómo obtener clientes satisfechos y leales, bajo una perspectiva latinoamericana. Bogotá: ECOE ediciones.

Vahos, J. (2014). Imagen Corporativa: modelos de gestión. Antioquía: Editorial Universidad Pontificia Bolivariana.

Vieites, R. (2012): Atraer y fidelizar clientes. Santiago de Compostela, España: C.E.E.I Galicia, S.A. Recuperado de http://datos.portaldelcomerciante.com/MiAfic/userfiles/167/Biblioteca/3dad1d4ec4fee5f7c719Guiapara-atraer-Fidelizar-Clientes_cas.pdf 


\section{RLCS, Revista Latina de Comunicación Social, 72 - Páginas 751 a 764 Investigación | DOI: 10.4185/RLCS, 72-2017-1190| ISSN 1138-5820 | Año 2017}

Varela, R. (2008). Innovación empresarial: arte y ciencia en la creación de empresas. Bogotá, Colombia: Pearson Educación de Colombia Cia. Ltda.

\section{Cómo citar este artículo / Referencia normalizada}

VK Duque Rengel, ME Abendaño Ramírez, AV Velásquez Benavides (2017): “Análisis de los factores de comunicación que inciden en la fidelización de los públicos universitarios". Revista Latina de Comunicación Social, 72 , pp. 751 a 764.

http://www.revistalatinacs.org/072paper/1190/40es.html

DOI: $\underline{10.4185 / R L C S-2017-1190}$

\section{- En el interior de un texto:}

... VK Duque Rengel, ME Abendaño Ramírez, AV Velásquez Benavides (2017: 751 a 764)... o

... VK Duque Rengel et al, 2017 (751 a 764)...

Artículo recibido el 18 de diciembre de 2016. Aceptado el 25 de junio.

Publicado el 30 de junio de 2017 